\title{
Renal NKCC2 Is Dual Regulated by the Synergy of 20-HETE and High-Fat Diet in CYP4F2 Transgenic Mice
}

\author{
Jingjing $\mathrm{Wu}^{\mathrm{a}}$ Guangrui Lai ${ }^{\mathrm{b}}$ Fangjie Chen $^{\mathrm{a}}$ Bijun Zhang ${ }^{\mathrm{b}}$ Yanyan Zhao $^{\mathrm{a}}$ \\ ${ }^{a}$ Department of Medical Genetics, School of Life Sciences, China Medical University, Shenyang, China; ${ }^{\text {bDepartment }}$ \\ of Clinical Genetics, Shengjing Hospital of China Medical University, Shenyang, China
}

\section{Keywords}

20-Hydroxyeicosatetraenoic acid $\cdot \mathrm{Na}^{+}-\mathrm{K}^{+}-2 \mathrm{Cl}^{-}$

cotransporter $\cdot$ High-fat diet - Obesity-related hypertension

\begin{abstract}
Introduction: 20 -Hydroxyeicosatetraenoic acid (20-HETE) is the metabolite of cytochrome P450, which modulates blood pressure by inhibiting renal sodium transport. However, the molecular mechanisms underlying the role of 20-HETE in the development of obesity-related hypertension remain unclear, necessitating this study. Methods: Cytochrome P450 4F2 (CYP4F2) transgenic mice fed high-fat diet (HFD) were used as research animal models. The expression of renal ion transport molecules targeted by 20 -HETE was evaluated by real-time $P C R$ and Western blot (WB). The regulatory effect of $20-\mathrm{HETE}$ and $\mathrm{HFD}$ on renal $\mathrm{Na}^{+}-\mathrm{K}^{+}-2 \mathrm{Cl}^{-}$cotransporter, isoform 2 (NKCC2) was explored by immunoprecipitation, WB, and luciferase assay. Results: A 2-week HFD feeding dramatically decreased protein abundance but increased renal NKCC2 mRNA expression in CYP4F2 transgenic mice. The decrease in NKCC2 protein was demonstrated to be due to ubiquitination induced by the synergy between 20-HETE and HFD. The increased PPAR- $\gamma$ protein in CYP4F2 transgenic mice fed HFD and the activation of rosiglitazone on the luciferase reporter construct of the NKCC2 promoter demon-
\end{abstract}

strated that the increase in NKCC2 mRNA in CYP4F2 transgenic mice fed HFD was a consequence of elevated PPAR- $\gamma$ protein induced by the synergy between 20-HETE and HFD. Conclusions: Our data demonstrated that the synergy between 20-HETE and HFD could decrease NKCC2 protein via posttranslational ubiquitination, which was thought to be the main mechanism underlying the short-term effect in response to HFD and might be responsible for the adaptive modulation of renal NKCC2 to resist sodium retention. Moreover, the increased NKCC2 mRNA expression via PPAR- $\gamma$ induced transcriptional regulation was thought to be the main mechanism underlying the long-term effect in response to HFD and plays a pivotal role in the development of obesity-related hypertension. @ 2021 The Author(s).

Published by S. Karger AG, Basel

\section{Introduction}

Obesity has become a global pandemic since it is a major risk factor for metabolic syndrome, which is associated with insulin resistance, dyslipidemia, and hypertension. However, the mechanisms by which obesity induces hypertension are not well understood. Structural analysis of the kidneys shows obese humans and obese animal karger@karger.com www.karger.com/kbr

Karger $\stackrel{\text { ' }}{5}$

GOPEN ACCESS
(C) 2021 The Author(s)

Published by S. Karger AG, Basel

This is an Open Access article licensed under the Creative Commons Attribution-NonCommercial-4.0 International License (CC BY-NC) (http://www.karger.com/Services/OpenAccessLicense), applicable to the online version of the article only. Usage and distribution for commercial purposes requires written permission.
Correspondence to:

Yanyan Zhao, zhaoyy@cmu.edu.cn 
models all have obesity-related renal damage [1], suggesting that renal injury due to excessive body weight might contributes to the elevation of blood pressure. In the kidney, the biochemical milieu of dyslipidemia, hormonal abnormalities, inflammation, and hyperinsulinemia, which is common in obese patients, has been associated with disruptions to fluid and electrolyte balance. Therefore, obesity-induced hypertension may result from an elevation in circulating volume due to increased renal sodium reabsorption in the proximal tubule [2-4], thick ascending limb [4-7], and/or distal tubule [4-7].

20 -Hydroxyeicosatetraenoic acid (20-HETE) is a potent vasoconstrictor that regulates the vascular tone in arterioles by blocking $\mathrm{Ca}^{2+}$-activated $\mathrm{K}^{+}$channels. It also inhibits sodium reabsorption in the proximal tubule and thick ascending limb of Henle's loop by inhibiting the activities of $\mathrm{Na}^{+}-\mathrm{K}^{+}$-ATPase, $\mathrm{Na}^{+} / \mathrm{H}^{+}$exchanger (NHE3), 70-pS $\mathrm{K}^{+}$channel (ROMK), and $\mathrm{Na}^{+}-\mathrm{K}^{+}-2 \mathrm{Cl}^{-}$cotransporter, isoform 2 (SLC12A1; NKCC2) $[8,9]$. Recently, Savas et al. [10] found that 20-HETE is able to increase the abundance of renal sodium chloride cotransporter (SLC12A3; NCC) in the distal convoluted tubule. It appears that the impact of excessive body weight and high levels of 20-HETE converges on nephrons involved in the modulation of blood pressure. Therefore, we speculated that 20-HETE plays a role in obesity-related hypertension.

Cytochrome P450 4F2 (CYP4F2) encodes an $\omega$-hydroxylase that catalyzes the arachidonic acid to 20HETE, and is responsible for the majority of 20-HETE synthesis in the human kidney and liver [9]. We previously generated a CYP4F2 transgenic mouse model and observed that 20-HETE in the kidney exerts a dual role in the modulation of blood pressure; under normal diet conditions, 20-HETE contributes to the elevation of blood pressure [11], whereas under high-salt diet conditions, 20-HETE induces natriuresis [12].

It is widely recognized that PPAR- $\gamma$ is a nutrient-sensing molecule and plays an important role in the regulation of fatty acid/lipid metabolism; however, it is also associated with fluid retention and edema. PPAR- $\gamma$-induced fluid retention and edema formation have been proposed to increase vascular permeability [13-15] and vasodilation [16] and to exert effects on the kidney. The latter is likely to elevate sodium and water reabsorption, possibly in response to a decrease in mean arterial blood pressure and/or a direct effect of PPAR- $\gamma$. We speculate that PPAR- $\gamma$ might play an important role in obesity-induced hypertension.
Obesity-induced hypertension is more nuanced than salt-related hypertension, and the mechanisms remain ambiguous. Furthermore, studies on several animal models have shown that obesity resulting from a high-fat diet (HFD) affects the expression of cytochrome P450 enzymes [17-24]. In this study, we aimed to investigate the effect of 20-HETE on renal natriuresis in obesity-related hypertension by feeding CYP4F2 transgenic mice HFD for 2 weeks.

\section{Materials and Methods}

\section{Animal Studies}

The CYP4F2 transgenic mice overexpressing CYP4F2 was a FVB strain [11]. Transgenic mice were identified by PCR using DNA isolated from tail biopsies with primers described previously. Mice were housed in pathogen-free facilities under a 12-h lightdark cycle system. Experiments were performed on 12- to 16-weekold male transgenic mice weighing between 24 and $30 \mathrm{~g}$. All mice were weight- and age-matched with wild-type (WT) mice as controls. Mice had free access to water at all times and were fed with normal diet or HFD ( $60 \%$ kcal fat) for 2 weeks $(n=4)$. All animal experimental protocols were conducted in accordance with the National Institutes of Health Guide for the Care and Use of Laboratory Animals (NIH Publications no. 8023, revised 1978).

\section{Real-Time PCR}

Total RNA was extracted from the kidneys using TRIzol reagent (Invitrogen, Carlsbad, CA, USA) and reverse transcribed into cDNA with the reverse transcription reagent kit (Promega, Madison, WI, USA). Real-time PCR was performed on the ABI 7500 system (Applied Biosystems, Foster City, CA, USA) in a 20$\mu \mathrm{L}$ SYBR green PCR containing $1 \times$ SYBR green PCR master mix (Applied Biosystems, Foster City, CA, USA), 10 ng cDNA, and 100 nM forward and reverse primers. The sequences of used primers are shown in Table 1.

Samples were subjected to 40 cycles of 2 temperature steps as follows: $95^{\circ} \mathrm{C}$ for $15 \mathrm{~s}, 60^{\circ} \mathrm{C}$ for $1 \mathrm{~min}$. Dissociation curves were generated to ensure that a single and specific product was amplified. Cycle threshold values were analyzed by SDS 2.4 software (Applied Biosystems, Foster City, CA, USA), and relative quantification of $\mathrm{Na}^{+}-\mathrm{K}^{+}$-ATPase, NHE3, ROMK, NKCC2, NCC, as well as Cyp4a10, Cyp4a12, and Cyp4a14 expression was determined using the comparative cycle threshold method with the GAPDH transcript as an internal control.

\section{Immunoprecipitation and Western Blot}

Renal protein samples were prepared by homogenizing the frozen tissues in lysis buffer containing protease inhibitors, and the concentration was determined by the Bradford method. The protein samples were preincubated with primary antibody by rotating at $4^{\circ} \mathrm{C}$ overnight, followed by the addition of $20 \mu \mathrm{L}$ equivalents of protein A/G PLUS-agarose (Santa Cruz Biotechnology, Santa Cruz, CA, USA) and rotating for $1 \mathrm{~h}$. Protein A/G beads were collected and washed with lysis buffer 3 times. Immunoprecipitates were resolved by SDS-polyacrylamide gel electrophoresis and analyzed by Western blot (WB). In WB analysis, the protein samples 
Table 1. Primers for quantitative PCR

\begin{tabular}{|c|c|}
\hline Name & Sequence \\
\hline $\mathrm{Na}^{+}-\mathrm{K}^{+}$-ATPase-forward & 5'-CTCCAGCAACAGGACCCGGCG-3' \\
\hline $\mathrm{Na}^{+}-\mathrm{K}^{+}$-ATPase-reverse & 5'-GATCTCAGCGGCCCTTGCAGG-3' \\
\hline NHE3-forward & 5'-AAGCTGTACAAGAGGGAGCG-3' \\
\hline NHE3-reverse & 5'-TGATGGTGTAGTTGTGTGCC-3' \\
\hline ROMK-forward & 5'-CATCATTGTGAGGCTTAAGATTCATT-3' \\
\hline ROMK-reverse & 5'-GCTGTTCAGTTTTGTCTGTGCAA-3' \\
\hline NCC-forward & 5'-CTTCGGCCACTGGCATTCTG-3' \\
\hline NCC-reverse & 5'-GATGGCAAGGTAGGAGATGG-3' \\
\hline NKCC2-forward & 5'- GAGATTGGCGTGGTCATAGTCAGAA-3' \\
\hline NKCC2-reverse & 5'-TGCTGCTGATGTTGCCGTCTTT-3' \\
\hline Cyp4a10-forward & 5'-TTCCCTGATGGACGCTCTTTA-3' \\
\hline Cyp4a10-reverse & 5'-GCAAACCTGGAAGGGTCAAAC-3' \\
\hline Cyp4a12-forward & 5'-CCAGCATTACACGAACAGAGT-3' \\
\hline Cyp4a12-reverse & 5'-GATTTCTAGCTCCCTGGATTGG-3' \\
\hline Cyp4a14-forward & 5'-GACTCTTGGGACAATGGACA-3' \\
\hline Cyp4a14-reverse & 5'-AGTTCCTTCCTCTGGCTGGT3' \\
\hline GAPDH-forward & 5'-TAACATCAAATGGGGTGAGG-3' \\
\hline GAPDH-reverse & 5'-GGTTCACACCCATCACAAAC-3' \\
\hline
\end{tabular}

were subjected to 8 or $10 \%$ SDS-polyacrylamide gel electrophoresis and transferred onto polyvinylidene difluoride (Millipore, Lake Placid, NY, USA) membranes. The membranes were blocked with $5 \%$ nonfat dry milk in TBS containing $0.1 \%$ Tween-20 and incubated with primary antibodies and then with horseradish peroxidase-conjugated secondary antibodies, according to the manufacturer's instructions. WB analysis was visualized with the enhanced chemiluminescence kit obtained from Thermo Scientific. Primary antibodies included $\mathrm{Na}^{+}-\mathrm{K}^{+}$-ATPase (Cell Signaling Technology, Danvers, MA, USA), NHE3 (Abcam, Cambridge, MA, USA), ROMK (Alomone Labs, Jerusalem, Israel), NCC (Upstate Biotech Millipore, Lake Placid, NY, USA), NKCC2 (Upstate Biotech Millipore, Lake Placid, NY, USA), ubiquitin (Cell Signaling Technology, Danvers, MA, USA), Nedd4-2 (Abcam, Cambridge, MA, USA), PPAR- $\gamma$ (Proteintech Group, Chicago, IL, USA), and GAPDH (Proteintech Group, Chicago, IL, USA).

\section{Plasmid Construction}

A 1,569-bp murine NKCC2 promoter linked to the luciferase reporter gene has been constructed. The luciferase reporter construct of NKCC2 was generated with the forward primer $5^{\prime}$-CGGCAGAACCTTACAACCG- $3^{\prime}$ and the reverse primer $5^{\prime}$-ACTTACCGCCCCATCCAG- $3^{\prime}$. All constructs were confirmed by sequencing with no coding frame shift in the luciferase gene. For transient transfection, the plasmids were purified using a Qiagen Plasmid Midi kit (Qiagen, Valencia, CA, USA).

\section{Cell Culture and Transient Transfection}

HEK-293T cells were maintained in Dulbecco's modified Eagle's medium supplemented with $10 \%$ fetal bovine serum, $100 \mathrm{U} /$ $\mathrm{mL}$ of penicillin, $100 \mu \mathrm{g} / \mathrm{mL}$ of streptomycin, and $5 \mu \mathrm{g} / \mathrm{mL}$ M-Plasmocin (Invivogen, San Diego, CA, USA) at $37^{\circ} \mathrm{C}$ in a humidified atmosphere containing 5\% $\mathrm{CO}_{2}$. HEK-293T cells were subcultured into 24 well plates, grown to $80-90 \%$ confluence after $24 \mathrm{~h}$, and transiently transfected using Lipofectamine TM3000, following the manufacturer's protocol, with $0.8 \mu \mathrm{g}$ of plasmid DNA and 0.01

Regulation of NKCC2 by 20-HETE and High-Fat Diet $\mu \mathrm{g}$ of pRL-TK encoding for Renilla luciferase, which was used to normalize transfection efficiency. Twenty-four hours after transfection, treated HEK-293T cells with PPAR- $\gamma$ agonist rosiglitazone (RGZ) (MCE, New Jersey, USA) at the final concentration of 0, 10, $50,100,200 \mathrm{nM}$ for $24 \mathrm{~h}$, the cells were harvested, and the luciferase activities were measured using the dual-luciferase reporter assay system (Promega, Madison, WI, USA) and a Lumat LB 9507 luminometer (Bethold Technologies, Bad Wildbad, Germany).

\section{Measurement of Blood Pressure}

The blood pressure of experimental mice was measured by the tail-cuff method, using the IITC Life Science Model 1631 tail pulse detection system (IITC Life Science, USA) according to the manufacturer's instructions. After 5 days of training, blood pressure was recorded from conscious mice that were fed with either a normal diet or a HFD. One measurement session involved 8 repetitions, and at least 3 sessions were performed on each mouse to calculate the average.

\section{Measurement of Plasma Glucose}

The blood samples from experimental mice fasted overnight were obtained by enucleating eyeballs, and the plasma was isolated by centrifuging. Plasma glucose was determined by the oxidaseperoxidase method (R\&D Systems, Minneapolis, MN, USA).

\section{Result}

\section{Physiological Assessment of CYP4F2 Transgenic Mice}

Fed HFD for 2 Weeks

We compared the systolic blood pressure, fasting plasma glucose, and body weight of mice in each group. Under normal diet conditions, as shown in Figure $1 \mathrm{~A}$ and $\mathrm{B}$, systolic blood pressure (129.9 $\pm 5.78 \mathrm{~mm} \mathrm{Hg}$ ) and fasting 


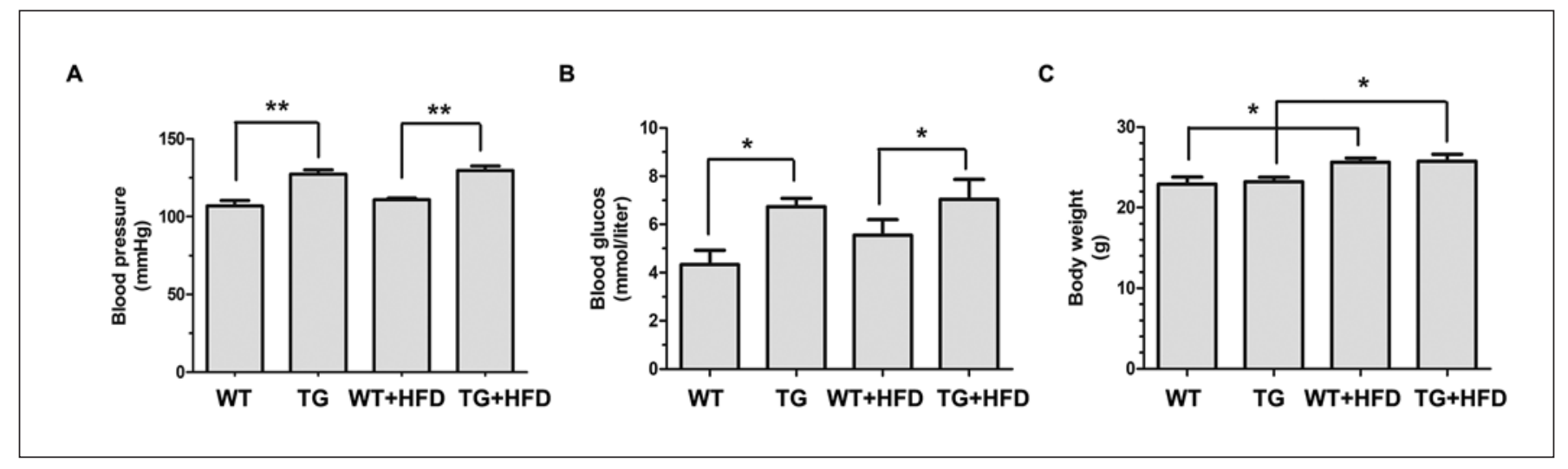

Fig. 1. Effect of 20-HETE and HFD on mice blood pressure, blood glucose, and body weight. Blood pressure (A), blood glucose (B), and body weight (C) were measured from WT and TG mice fed either normal diet or HFD for 2 weeks $(n=4)$. The experiments were performed 3 times independently. ${ }^{*} p<0.05,{ }^{* *} p<0.01$ compared with the corresponding group. WT, wild-type mice; TG, transgenic mice; 20-HETE, 20-hydroxyeicosatetraenoic acid; HFD, high-fat diet.

plasma glucose $(7.63 \pm 2.06 \mathrm{mmol} / \mathrm{L})$ were significantly increased in CYP4F2 transgenic mice compared to those in WT mice $(105.53 \pm 6.698 \mathrm{~mm} \mathrm{Hg}$ and $4.04 \pm 1.97$ $\mathrm{mmol} / \mathrm{L}$ for systolic blood pressure and fasting plasma glucose, respectively) due to overproduction of 20-HETE as reported previously [25]. Body weight (Fig. 1C) showed no difference between CYP4F2 transgenic and WT mice. Two weeks of HFD feeding increased body weight in both genotypes, about $11.02 \pm 6.11 \%$ in CYP4F2 transgenic mice and $10.35 \pm 5.41 \%$ in WT mice compared to that in normal diet fed mice. After 2 weeks of HFD feeding, systolic blood pressure and fasting plasma glucose remained unchanged in CYP4F2 transgenic and WT mice.

\section{Screening the Expression of Renal Ion Transport}

Molecules Targeted by 20-HETE in CYP4F2

Transgenic Mice Fed HFD

We detected the expression of renal ion transport molecules targeted by 20 -HETE, including $\mathrm{Na}^{+}-\mathrm{K}^{+}$-ATPase, NHE3, ROMK, NKCC2, and NCC. Figure 2 shows the results of WB and real-time PCR from WT and CYP4F2 transgenic mice fed either normal diet or HFD.

Under normal diet conditions, $\mathrm{Na}^{+}-\mathrm{K}^{+}$-ATPase expression was lower in CYP4F2 transgenic mice than in WT mice, but mRNA analysis showed no differences between the 2 groups (Fig. 2A). HFD moderately, but not significantly, decreased the protein expression of $\mathrm{Na}^{+}$$\mathrm{K}^{+}$-ATPase in WT mice and CYP4F2 transgenic mice, and $\mathrm{Na}^{+}-\mathrm{K}^{+}$-ATPase mRNA was substantially increased in both mouse groups.
No differences were observed in the protein and mRNA expression of NHE3 and ROMK under normal diet conditions between WT and CYP4F2 transgenic mice. After a 2-week HFD feeding, the protein abundance of NHE3 and ROMK remained unchanged in both WT and CYP4F2 transgenic mice. However, we found that NHE3 and ROMK mRNA were obviously elevated in response to HFD in both WT and CYP4F2 transgenic mice (Fig. 2B, C).

Compared with those in WT mice, NKCC2 protein and mRNA levels in CYP4F2 transgenic mice were reduced under normal diet conditions (Fig. 2D). A dramatic reduction in NKCC2 protein was detected in both WT and CYP4F2 transgenic mice fed HFD. After 2 weeks of HFD feeding, there was a significant increase in NKCC2 mRNA in both WT and transgenic mouse groups. Moreover, surprisingly, NKCC2 mRNA in transgenic mice was dramatically higher than that in WT mice under HFD conditions, and thus we speculated that 20-HETE synergizes with HFD to increase renal NKCC2 mRNA expression.

NCC is another member of the SLC12a family transporter. Under normal diet conditions, there was a trend of higher NCC protein $(p=0.089)$ and mRNA $(p=0.091)$ levels in CYP4F2 transgenic mice than in WT mice (Fig. 2E), but there was no statistical significance. HFD dramatically increased the protein abundance of NCC by approximately $140.3 \%$ in CYP4F2 transgenic mice and $127.3 \%$ in WT mice. Moreover, under HFD conditions, CYP4F2 transgenic mice had a significantly higher renal 


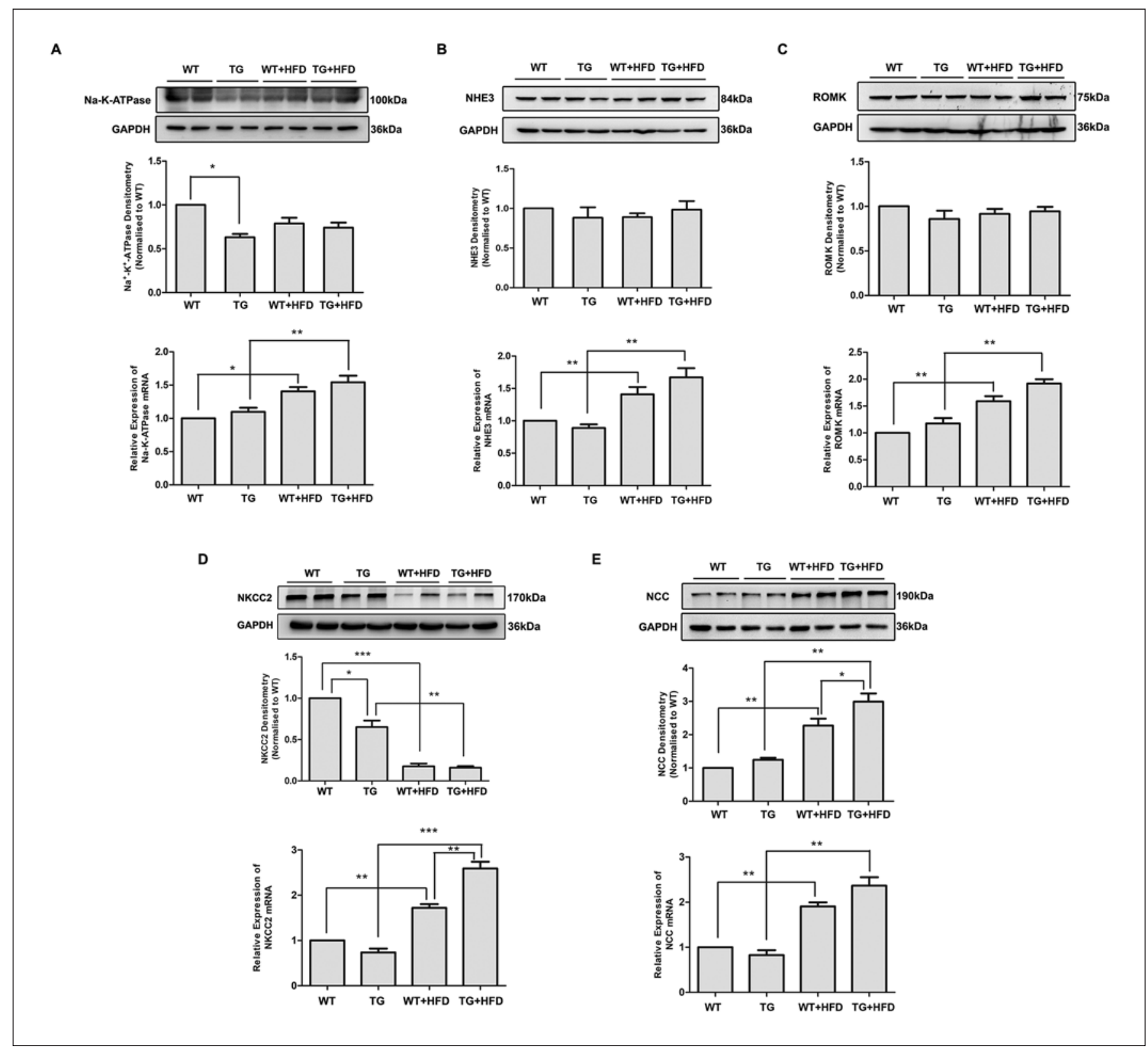

Fig. 2. Effect of 20-HETE and HFD on renal $\mathrm{Na}^{+}-\mathrm{K}^{+}$-ATPase, NHE3, ROMK, NKCC2, and NCC expression. Renal $\mathrm{Na}^{+}-\mathrm{K}^{+}-$ ATPase (A), NHE3 (B), ROMK (C), and NKCC2 (D), NCC protein, and mRNA expression (E) was detected by WB and real-time PCR from WT and TG mice fed either normal diet or HFD for 2 weeks

NCC level than WT mice. Furthermore, the mRNA expression of NCC in response to HFD was consistent with changes in its protein expression. $(n=4)$. The experiments were performed 3 times independently. ${ }^{*} p<0.05,{ }^{* *} p<0.01,{ }^{* * *} p<0.001$ compared with the corresponding group. WT, wild-type mice; TG, transgenic mice; 20-HETE, 20-hydroxyeicosatetraenoic acid; HFD, high-fat diet; WB, Western blot.
Synergistic Effect of 20-HETE and HFD on the Downregulation of NKCC2 through Posttranslational Ubiquitination

As NKCC2 mRNA expression was higher in transgenic mice under HFD conditions, we investigated whether 


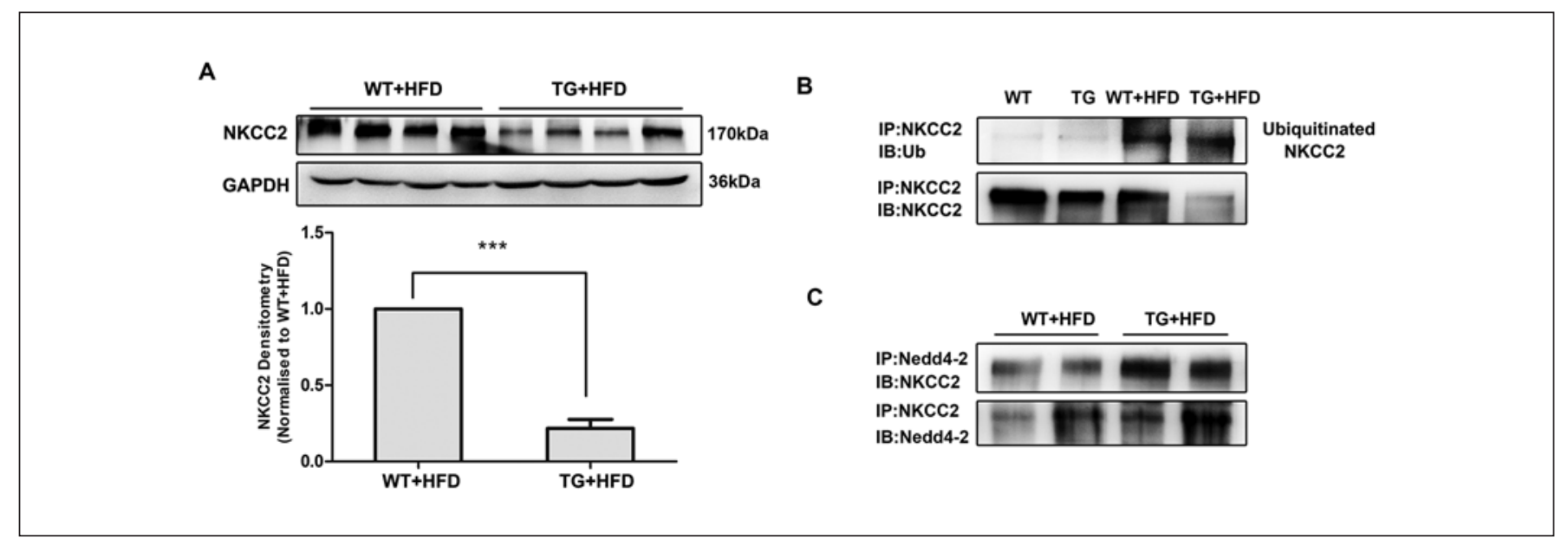

Fig. 3. Effect of 20-HETE and HFD on renal NKCC2 protein ubiquitination. A NKCC2 protein and mRNA expression from WT and TG mice fed HFD was detected by WB and real-time PCR $(n=4)$. B Ubiquitinated NKCC2 protein from the kidney of WT and TG mice fed with either normal diet or HFD was detected by IP with NKCC2 antibody, followed by WB with ubiquitin or NKCC2 antibody $(n=4)$. C The interaction of NKCC2 and Nedd4-2 in the

this difference was also apparent at the protein level by comparing the NKCC2 protein abundance between $C Y$ P4F2 transgenic and WT mice fed HFD (Fig. 3A). Densitometry analysis identified that under HFD conditions, renal NKCC2 was reduced by approximately $78 \%$ in $C Y$ P4F2 transgenic mice compared with that in WT mice, suggesting that HFD and 20-HETE synergize to reduce renal NKCC2 expression.

Here, we found that 20-HETE and HFD increased the mRNA expression but decreased the protein expression of NKCC2 in CYP4F2 transgenic mice, and therefore speculate that the mechanism for decreasing NKCC2 protein expression is a result of posttranslational modification. Protein ubiquitination is one of the most important and widely available protein posttranslational modifications.

To investigate whether the posttranslational modification of NKCC2 was a result of ubiquitination, we performed immunoprecipitation (IP) experiments to examine the ubiquitination level of NKCC2. As shown in Figure $3 \mathrm{~B}$, ubiquitination of NKCC2 was induced by HFD, and the ubiquitination level was significantly higher in CYP4F2 transgenic mice than in WT mice. Furthermore, an interaction between NKCC2 and Nedd4-2 was detected, and we observed that CYP4F2 transgenic mice had increased IP compared to that in WT mice (Fig. 3C). These observations suggested that HFD may induce ubiq- kidney of WT and TG mice fed with HFD was detected by co-IP: IP with NKCC2 or Nedd4-2 antibody, followed by WB with Nedd4-2 or NKCC2 antibody $(n=4)$. The experiments were performed 3 times independently. ${ }^{* * *} p<0.001$ compared with the corresponding group. WT, wild-type mice; TG, transgenic mice; 20-HETE, 20-hydroxyeicosatetraenoic acid; HFD, high-fat diet; IP, immunoprecipitation; WB, Western blot.

uitination of NKCC2 via Nedd4-2 as a ubiquitin E3 ligase. More importantly, 20-HETE can synergistically enhance the ubiquitination level of NKCC2.

\section{Synergistic Effect of 20-HETE and HFD on the}

Upregulation of NKCC2 through PPAR- $\gamma$-Mediated

Transcriptional Regulation

PPAR- $\gamma$ is an important transcriptional factor belonging to the ligand-activated nuclear receptor superfamily and is mainly involved in regulating lipid metabolism, insulin sensitivity, and glucose homeostasis. We detected the expression of PPAR- $\gamma$ protein in the kidneys of $C Y$ P4F2 transgenic mice fed HFD and compared it to that in the normal diet group. HFD increased the expression of PPAR- $\gamma$ (Fig. 4A). Moreover, PPAR- $\gamma$ and NKCC2 mRNA expression was significantly higher in CYP4F2 transgenic mice than in WT mice. This suggests that PPAR- $\gamma$ is induced synergistically by 20 -HETE and HFD, and likely mediates transcriptional regulation of renal NKCC2 gene in response to HFD in CYP4F2 transgenic mice.

To confirm our hypothesis, a luciferase reporter construct containing a 1,569-bp promoter of the mouse NKCC2 gene was generated and transiently transfected into HEK-293T cells. Treatment with RGZ, a PPAR- $\gamma$ agonist, increased the transcriptional activity of NKCC2 in a dose-dependent manner (Fig. 4B). The transcriptional 
A
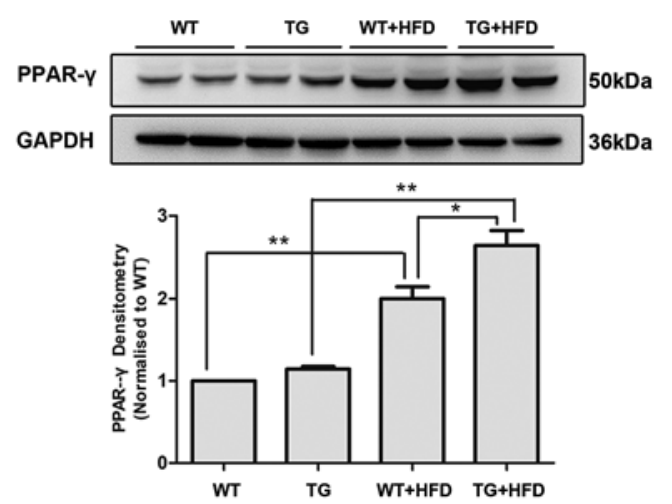

B

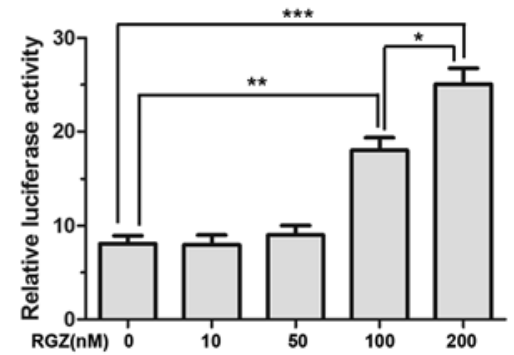

Fig. 4. $P P A R-\gamma$ mediated the transcriptional regulation on renal NKCC2 in CYP4F2 transgenic mice with HFD. A Renal PPAR- $\gamma$ protein expression was detected by WB from WT and TG mice fed normal diet or HFD for 2 weeks $(n=4)$. B The transcriptional activity of NKCC2 was analyzed by relative luciferase activities of NKCC2 promoter luciferase reporter constructs treated with vari- ous PPAR- $\gamma$ agonist RGZ concentrations from 0 to $200 \mathrm{nM}$ in HEK-293T cells. The experiments were performed 3 times independently. ${ }^{*} p<0.05,{ }^{* *} p<0.01,{ }^{* * *} p<0.001$ compared with the corresponding group. WT, wild-type mice; TG, transgenic mice; HFD, high-fat diet; WB, Western blot; RGZ, rosiglitazone.
A

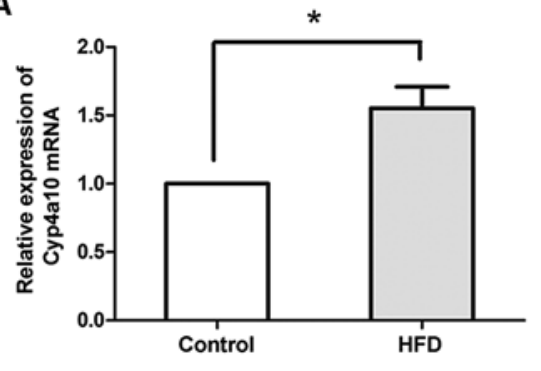

B

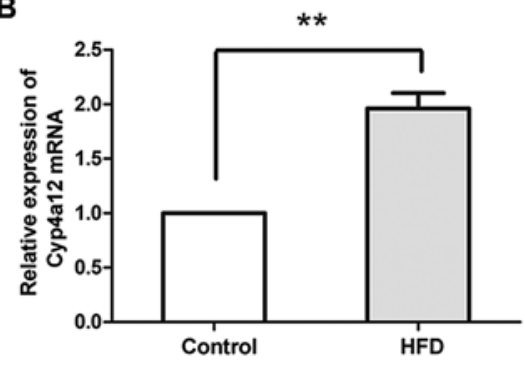

C

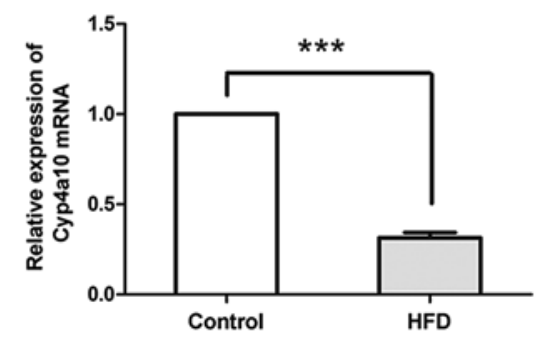

Fig. 5. Effect of HFD on renal Cyp4a10, Cyp4a12, and Cyp4a14 expression. Renal Cyp4a10 (A), Cyp4a12 (B), and Cyp4a14 mRNA expression $(\mathbf{C})$ was detected by real-time PCR from WT mice fed
HFD for 2 weeks $(n=4)$. The experiments were performed 3 times independently. ${ }^{*} p<0.05,{ }^{* *} p<0.01,{ }^{* * *} p<0.001$ compared with the corresponding group. WT, wild-type mice; HFD, high-fat diet. activity increased by 122.3 and $209.6 \%$ in the control at RGZ concentrations of 100 and $200 \mathrm{nM}$, respectively. These findings demonstrated that PPAR $-\gamma$ could mediate transcriptional regulation of the NKCC2 gene. Therefore, 20-HETE possibly synergizes with HFD to elevate NKCC2 gene expression through PPAR- $\gamma$-mediated transcriptional regulation.

\section{Effect of HFD on Renal Cyp4a Isoform Expression}

To identify the effect of HFD on renal Cyp4a isoforms, we detected the mRNA expression of Cyp4a subfamily members expressed in mouse kidneys, including $\mathrm{Cy}$ - p4a10, Cyp4a12, and Cyp4a14. As shown in Figure 5, after 2 weeks of HFD feeding, the mRNA expression of $C y$ p $4 a 10$ and Cyp4a12 was significantly increased by 55.27 and $96.05 \%$, respectively, whereas that of Cyp4a14 was dramatically decreased by $68.56 \%$.

\section{Discussion/Conclusion}

HFD and abnormal 20-HETE levels are involved in the development of obesity-induced hypertension. In the present study, we found that 20-HETE affects renal 
NKCC2 expression in CYP4F2 transgenic mice fed HFD. A combination of 20-HETE and HFD increased renal NKCC2 mRNA expression through PPAR- $\gamma$-mediated transcriptional regulation but decreased renal NKCC2 protein abundance through ubiquitination. Furthermore, activation of the PPAR- $\gamma$ agonist RGZ on the luciferase reporter construct of the NKCC2 promoter provides evidence that PPAR- $\gamma$ may regulate NKCC2 gene expression. To our knowledge, this is the first study reporting that 20-HETE and HFD synergistically play a dual regulatory role in renal NKCC2 expression. Based on this mechanism, we speculate that the synergic modulatory effect of 20-HETE and HFD on renal NKCC2, ubiquitination, and PPAR- $\gamma$ signaling may in turn play a dominant role in various feeding periods.

Obesity is an important health threat associated with cardiovascular morbidity, while the exact mechanisms remain unclear. The imbalance of fluids and electrolytes may be a consequence of obesity that directly impacts blood pressure. Obesity induced by HFD reliably alters renal complement components, microenvironment, and structure $[1,3,5,7,26,27]$. Previously, we generated $C Y$ $P 4 F 2$ transgenic mice to explore the effect of 20-HETE on blood pressure modulation and demonstrated that 20HETE plays a dual role in the modulation of blood pressure under various conditions $[11,12]$. The aim of this study was to investigate the role of 20-HETE in watersodium metabolism after short-term HFD feeding. We observed that after a 2-week HFD feeding, high levels of 20-HETE synergized with HFD to dramatically reduce renal NKCC2 protein expression, while significantly enhancing NKCC2 mRNA expression. These seemingly contradictory findings provide evidence that in the 2-week HFD condition, posttranslational ubiquitination plays a pivotal role in the regulation of NKCC2 protein expression, and we employed IP to examine the ubiquitination level of NKCC2, which confirmed our speculation. Additionally, we observed a substantial interaction between NKCC2 and Nedd4-2, which was higher in $C Y$ P4F2 transgenic mice than in WT controls. Taken together, these data indicate that after a 2-week HFD feeding, ubiquitination plays an important role in the modulation of NKCC2 expression to resist sodium retention, and 20HETE can facilitate this action possibly because of its role in natriuresis.

This adaptable metabolic mechanism of organisms to HFD can also be observed in the changes in the expression of renal Cyp4a isoforms after HFD feeding. HFD significantly increased the mRNA expression of Cyp4a12, which is the predominant 20-HETE synthase in the mouse kidney [28-32], whereas Cyp4a14, which has been shown to suppress Cyp4a12 [30,33-35], exhibited significantly decreased mRNA expression. Zhou et al. [36] demonstrated that induction of renal 20-HETE by clofibrate can attenuate HFD-induced hypertension in rats. Therefore, we speculate that a 2 -week HFD feeding might result in elevated arachidonic acid $\omega$-hydroxylase activity and increased 20-HETE production, thereby playing renal modulation adaptation to HFD via 20-HETE natriuresis. Similarly, mRNA expression of Cyp4a10 was increased. Cyp $4 a 10$ plays an important role in the control of renal $\mathrm{Na}^{+}$transport and ultimately systemic blood pressure. However, a study on Cyp4a10 ${ }^{-1-}$ mice confirmed that Cyp4a10 did not contribute to 20-HETE production but was responsible for epoxygenase activity and EET synthesis [35], and EET, being analogous to 20HETE, plays a role in natriuresis in the kidney. These results suggested that under short-term HFD feeding, organisms can resist sodium retention induced by HFD through elevated 20-HETE and EET synthesis.

We found that NKCC2 mRNA was dramatically different between HFD and high-salt diet in CYP4F2 transgenic mice [12]. In high-salt diet conditions, almost no change was observed in NKCC2 mRNA expression in WT and CYP4F2 transgenic mice. Based on the different changes in NKCC 2 mRNA expression between HFD and high-salt diet, we postulate that there could be a mechanism involved in transcriptional regulation of NKCC2 in CYP4F2 transgenic mice under HFD conditions. We found that PPAR- $\gamma$ protein expression was markedly enhanced in response to HFD, especially in CYP4F2 transgenic mice, and correlated with an increase in NKCC2 mRNA. There is evidence that PPAR- $\gamma$ can affect the expression and/or activation of several renal water-sodium transporters and channel proteins to modulate renal water-sodium metabolism, but its role in NKCC2 regulation is still incompletely understood [16,37-43]. To delineate the molecular mechanism of elevated NKCC2 mRNA in response to HFD, we generated a luciferase reporter construct containing the promoter of mouse NKCC2 gene and treated it with RGZ, a synthetic agonist of PPAR- $\gamma$. RGZ increased the transcriptional activity of NKCC2 in a dose-dependent manner, suggesting that PPAR- $\gamma$ might directly modulate NKCC2 gene expression. These data suggest that the increase in NKCC2 mRNA in CYP4F2 transgenic mice fed HFD was a consequence of elevated PPAR- $\gamma$ protein induced by the synergy of 20 -HETE with HFD.

The distribution [43-45] and biological function [16, 44-48] of 20-HETE and PPAR- $\gamma$ overlapped; in theory, 
there may be a cross talk between 20-HETE and PPAR- $\gamma$. Some isoforms of the CYP450 subfamily are known to have PPRE in the promoter area of their genes; therefore, PPARs can modulate 20 -HETE production via transcriptional regulation of the CYP450 gene. Some studies have demonstrated that PPAR- $\gamma$ plays a biological role by increasing the production of 20-HETE [46, 49-52]. Additional reports have shown that 20-HETE is a potent activator of PPARs $[47,53,54]$. However, the relationship between 20-HETE and PPAR- $\gamma$ is still ambiguous. In the current study, the abundance of PPAR- $\gamma$ protein in WT and CYP4F2 transgenic mice with different 20-HETE levels under normal diet or HFD conditions demonstrated that 20-HETE exerts a modulatory effect on PPAR- $\gamma$ under HFD conditions in vivo. The CYP4F2 transgenic mouse model provides strong evidence for further study of the relationship between 20-HETE and PPAR- $\gamma$.

Increased tubular reabsorption of salt is important in the pathogenesis of obesity-related hypertension [2]. In the current study, the synergy of 20-HETE with a 2-week HFD feeding dramatically reduced renal NKCC2 protein expression. This is inconsistent with other studies on HFD animal models, which found that HFD enhanced the activity or protein levels of NKCC2 $[5,6]$. However, this result can also be echoed, to some extent, as the finding of a study by Power and colleagues [7] who examined the effect of HFD on C57BL/6 mice for 3 weeks. They found that HFD did not alter the expression of NKCC2 in the cortex or whole kidney as well as the phosphorylation of NKCC2 at S126. According to the results of the present study, the synergy between 20-HETE and HFD has a dual regulatory effect on NKCC2 expression through ubiquitination downregulation and transcriptional upregulation, which might explain the inconsistencies in NKCC2 expression in the kidney of mice fed HFD under diverse treatment periods from different laboratories.

We speculate that the feeding period of HFD was a crucial factor in the obtained results. The feeding periods in previous reports exceeded 3 weeks, whereas we employed 2 weeks. A possible explanation is that at a time period equal to or $<2$ weeks, the organism is able to adapt to HFD mainly via ubiquitination of NKCC2 protein during the early phase of NKCC2 expression. Decreased NKCC2 protein abundance was able to neutralize sodium retention due to HFD. Subsequently, in the next phase, the ubiquitination of NKCC2 protein was less pronounced, and the abundance of NKCC2 was normalized, resulting in NKCC2 failing to adjust itself to adapt to the internal environment, similar to that reported by Power and colleagues [7]. In the final phase, the abundance of

Regulation of NKCC2 by 20-HETE and

High-Fat Diet
NKCC2 protein gradually increased through transcriptional regulation of PPAR- $\gamma$, resulting in an increase in circulating volume, which, in turn, contributed to elevated blood pressure.

The underlying mechanism of obesity-related hypertension should be obesity, but not the effect of HFD per se. Similar but different obese models were examined during the same period, and renal NKCC2 protein abundance was found to be decreased, unchanged, or increased in obese Zucker rat or $\mathrm{db} / \mathrm{db}$ obese mouse models [4, 20, 55-59]. These data were different from those of HFDinduced obese animals. We presume that the difference in the final phenomenon between HFD-induced and genetically obese animal models was due to the different starting times of obesity. HFD-induced obese animal models were always generated after adulthood; however, genetically obese animal models showed onset of obesity generally since childhood. A great quantity of data have shown that the characteristics of pathophysiology, gene expression prolife, and epigenetic regulation in response to obesity differed between childhood obesity and adulthood obesity [60-64]. Further studies are required to understand the mechanisms leading to the diversity between HFD-induced and genetically obese animal models.

NCC mRNA was found to be significantly elevated after a 2-week HFD feeding, suggesting that increased transcription and/or stability of NCC mRNA was an important factor of the elevated NCC protein abundance. The potential molecular mechanism underlying the elevation of NCC expression in response to HFD has not been examined in the current study. However, it could be linked to the changes in hormones or fatty acids in response to HFD, but not PPAR- $\gamma$, because reports show that PPAR- $\gamma$ did not seem to have a regulatory effect on NCC $[39,65]$, and thus further studies are needed to investigate the underlying mechanism. A 2-week HFD feeding gives rise to different changes in the protein expression of NKCC2 and NCC, as well as $\mathrm{Na}^{+}-\mathrm{K}^{+}$-ATPase, NHE3, and ROMK. The outcome of the total function of these renal ion transporters may be 1 reason why there was no change in blood pressure in mice fed HFD for 2 weeks. At the same time, vasoconstriction caused by 20 -HETE was also the main reason why CYP4F2 transgenic mice were still hypertensive, despite NKCC2 downregulation.

In conclusion, in this study, we found that a synergy of 20-HETE with HFD resulted in dual regulation of renal NKCC2 in CYP4F2 transgenic mice. The synergistic effect between 20-HETE and HFD reduced NKCC2 protein abundance by ubiquitination via Nedd4-2 at the posttranslational level and enhanced NKCC2 mRNA expres- 
sion by inducing PPAR- $\gamma$ at the transcriptional level. From our observations and findings of other groups, we speculate that these 2 mechanisms might work in concert with HFD in CYP4F2 transgenic mice; however, these 2 mechanisms will take turns to play a dominant role at different stages. For a short-period HFD feeding, ubiquitination is the main modulation mechanism of renal NKCC2 to resist sodium retention and will exist for a short time only, while for a long-term HFD feeding, the transcriptional regulation of NKCC2 through PPAR- $\gamma$ signaling will play the main modulatory role, and could exist persistently, which may be an important cause of obesity-related hypertension.

\section{Statement of Ethics}

All animal experimental protocols were conducted in accordance with the National Institutes of Health Guide for the Care and Use of Laboratory Animals. All protocols were approved by the Animal Ethics Committee of China Medical University.

\section{Conflict of Interest Statement}

The authors declare that they have no conflicts of interest.

\section{Funding Sources}

This study was supported by the National Natural Science Foundation of China (NSFC 81400709) and Liaoning Provincial Natural Science Foundation of China (2019-MS-381).

\section{Author Contributions}

Guarantor of integrity of the entire study: Yanyan Zhao. Study concepts and design: Jingjing Wu. Definition of intellectual content: Jingjing $\mathrm{Wu}$ and Yanyan Zhao. Literature research: Jingjing Wu, Guangrui Lai, Fangjie Chen, Bijun Zhang, and Yanyan Zhao. Experimental studies: Jingjing $\mathrm{Wu}$ and Guangrui Lai. Data acquisition and analysis: Jingjing $\mathrm{Wu}$, Guangrui Lai, Fangjie Chen, and Bijun Zhang. Statistical analysis: Jingjing Wu and Guangrui Lai. Manuscript preparation: Jingjing Wu. Manuscript editing: Jingjing $\mathrm{Wu}$. Manuscript review: Jingjing $\mathrm{Wu}$ and Yanyan Zhao.

\section{References}

1 Serra A, Romero R, Lopez D, Navarro M, Esteve A, Perez N, et al. Renal injury in the extremely obese patients with normal renal function. Kidney Int. 2008 Apr;73(8):947-55.

2 DeMarco VG, Aroor AR, Sowers JR. The pathophysiology of hypertension in patients with obesity. Nat Rev Endocrinol. 2014 Jun; 10(6):364-76

3 Briffa J, Grinfeld E, Jenkin K, Mathai M, Poronnik $\mathrm{P}, \mathrm{McAinch} \mathrm{A}$, et al. Diet induced obesity in rats reduces $\mathrm{NHE} 3$ and $\mathrm{Na}+\mathrm{K}+-$ ATPase expression in the kidney. Clin Exp Pharmacol Physiol. 2015 Oct;42(10):111826.

4 Bickel CA, Verbalis JG, Knepper MA, Ecelbarger CA. Increased renal Na-K-ATPase, $\mathrm{NCC}$, and beta-ENaC abundance in obese Zucker rats. Am J Physiol Renal Physiol. 2001 Oct;281(4):F639-48.

5 Riazi S, Tiwari S, Sharma N, Rash A, Ecelbarger CM. Abundance of the $\mathrm{Na}-\mathrm{K}-2 \mathrm{Cl}$ cotransporter NKCC2 is increased by high-fat feeding in Fischer $344 \mathrm{X}$ Brown Norway (F1) rats. Am J Physiol Renal Physiol. 2009 Apr;296(4): F762-70.

6 Davies M, Fraser SA, Galic S, Choy SW, Katerelos M, Gleich K, et al. Novel mechanisms of $\mathrm{Na}+$ retention in obesity: phosphorylation of NKCC2 and regulation of SPAK/OSR1 by AMPK. Am J Physiol Renal Physiol. 2014 Jul 1;307(1):F96-F106.
7 Davies MR, Gleich K, Katerelos M, Lee M, Mount PF, Power DA. The thiazide-sensitive co-transporter promotes the development of sodium retention in mice with diet-induced obesity. Kidney Blood Press Res. 2015;40(5): 509-19.

8 Imig JD. 20-HETE or EETs: which arachidonic acid metabolite regulates proximal tubule transporters and contributes to pressure natriuresis? Am J Physiol Regul Integr Comp Physiol. 2004 Jul;287(1):R3-5.

9 Fan F, Roman RJ. Effect of cytochrome P450 metabolites of arachidonic acid in nephrology. J Am Soc Nephrol. 2017 Oct;28(10):284555.

10 Savas Ü, Wei S, Hsu M-H, Falck JR, Guengerich FP, Capdevila JH, et al. 20-Hydroxyeicosatetraenoic acid (HETE)-dependent hypertension in human cytochrome P450 (CYP) 4A11 transgenic mice: normalization of blood pressure by sodium restriction, hydrochlorothiazide, or blockade of the type 1 angiotensin II receptor. J Biol Chem. 2016 Aug 5;291(32): 16904-19.

11 Liu X, Zhao Y, Wang L, Yang X, Zheng Z, Zhang $\mathrm{Y}$, et al. Overexpression of cytochrome P450 4F2 in mice increases 20-hydroxyeicosatetraenoic acid production and arterial blood pressure. Kidney Int. 2009 Jun;75(12): 1288-96.

12 Wu J, Liu X, Lai G, Yang X, Wang L, Zhao Y. Synergistical effect of 20-HETE and high salt on NKCC2 protein and blood pressure via ubiquitin-proteasome pathway. Hum Genet. 2013 Feb;132(2):179-87.
13 Vasudevan AR, Balasubramanyam A. Thiazolidinediones: a review of their mechanisms of insulin sensitization, therapeutic potential, clinical efficacy, and tolerability. Diabetes Technol Ther. 2004 Dec;6(6):850-63.

$14 \mathrm{Ku} \mathrm{YH}$, Cho BJ, Kim MJ, Lim S, Park YJ, Jang $\mathrm{HC}$, et al. Rosiglitazone increases endothelial cell migration and vascular permeability through Akt phosphorylation. BMC Pharmacol Toxicol. 2017 Aug 30;18(1):62.

15 Sotiropoulos KB, Clermont A, Yasuda Y, Rask-Madsen C, Mastumoto M, Takahashi J, et al. Adipose-specific effect of rosiglitazone on vascular permeability and protein kinase $\mathrm{C}$ activation: novel mechanism for PPAR $\gamma$ agonist's effects on edema and weight gain. FASEB J. 2006 Jun;20(8):1203-5.

16 Kvandová M, Majzúnová M, Dovinová I. The role of PPARgamma in cardiovascular diseases. Physiol Res. 2016 Oct 24;65(Suppl 3): S343-63.

17 Knudsen JG, Bertholdt L, Gudiksen A, Gerbal-Chaloin S, Rasmussen MK. Skeletal muscle interleukin-6 regulates hepatic cytochrome P450 expression: effects of 16-week high-fat diet and exercise. Toxicol Sci. 2018 Mar 1;162(1):309-17.

18 Wang W, Yang J, Qi W, Yang H, Wang C, Tan $\mathrm{B}$, et al. Lipidomic profiling of high-fat dietinduced obesity in mice: importance of cytochrome P450-derived fatty acid epoxides. Obesity. 2017 Jan;25(1):132-40. 
19 Chiba T, Noji K, Shinozaki S, Suzuki S, Umegaki K, Shimokado K. Diet-induced non-alcoholic fatty liver disease affects expression of major cytochrome P450 genes in a mouse model. J Pharm Pharmacol. 2016 Dec;68(12):1567-76.

20 Park EC, Kim SI, Hong Y, Hwang JW, Cho GS, Cha HN, et al. Inhibition of CYP4A reduces hepatic endoplasmic reticulum stress and features of diabetes in mice. Gastroenterology. 2014 Oct;147(4):860-9.

21 Duan Y, Zhang F, Yuan W, Wei Y, Wei M, Zhou Y, et al. Hepatic cholesterol accumulation ascribed to the activation of ileum FxrFgf15 pathway inhibiting hepatic Cyp7al in high-fat diet-induced obesity rats. Life Sci. 2019 Sep 1;232:116638.

22 Jian T, Yu C, Ding X, Chen J, Li J, Zuo Y, et al. Hepatoprotective effect of seed coat of Euryale ferox extract in non-alcoholic fatty liver disease induced by high-fat diet in mice by increasing Rs-1 and inhibiting CYP2E1. J Oleo Sci. 2019;68(6):581-9.

23 Ding S, Qiu H, Huang J, Chen R, Zhang J, Huang B, et al. Activation of 20-HETE/ PPARs involved in reno-therapeutic effect of naringenin on diabetic nephropathy. Chem Biol Interact. 2019 Jun 6;307(6):116-24.

24 Sadler NC, Webb-Robertson BM, Clauss TR, Pounds JG, Corley R, Wright AT. High-fat diets alter the modulatory effects of xenobiotics on cytochrome P450 activities. Chem Res Toxicol. 2018 May 21;31(5):308-18.

25 Lai G, Wu J, Liu X, Zhao Y. 20-HETE induces hyperglycemia through the cAMP/PKAPhK-GP pathway. Mol Endocrinol. 2012 Nov; 26(11):1907-16.

26 Huang Y, Xu J, Wu X, Chen X, Bai X, Zhuang $Y$, et al. High expression of complement components in the kidneys of type 2 diabetic rats with diabetic nephropathy. Front Endocrinol. 2019 Jul 9;10:459.

27 Laurentius T, Raffetseder U, Fellner C, Kob R, Nourbakhsh M, Floege J, et al. High-fat dietinduced obesity causes an inflammatory microenvironment in the kidneys of aging LongEvans rats. J Inflamm. 2019 Jun 25;16:14.

28 Henderson CJ, Bammler T, Wolf CR. Deduced amino acid sequence of a murine cytochrome P-450 Cyp4a protein: developmental and hormonal regulation in liver and kidney. Biochim Biophys Acta. 1994 Jul 6;1200(2): 182-90.

29 Heng YM, Kuo CS, Jones PS, Savory R, Schulz RM, Tomlinson SR, et al. A novel murine P-450 gene, Cyp4a14, is part of a cluster of Cyp4a and Cyp4b, but not of CYP4F, genes in mouse and humans. Biochem J. 1997 Aug 1; 325(Pt 3):741-9.

30 Holla VR, Adas F, Imig JD, Zhao X, Price E Jr, Olsen $\mathrm{N}$, et al. Alterations in the regulation of androgen-sensitive Cyp 4a monooxygenases cause hypertension. Proc Natl Acad Sci U S A. 2001 Apr 24;98(9):5211-6.
31 Honeck H, Gross V, Erdmann B, Kärgel E, Neunaber R, Milia AF, et al. Cytochrome $\mathrm{P} 450$-dependent renal arachidonic acid metabolism in desoxycorticosterone acetate-salt hypertensive mice. Hypertension. 2000 Oct; 36(4):610-6.

32 Stec DE, Flasch A, Roman RJ, White JA. Distribution of cytochrome P-450 $4 \mathrm{~A}$ and $4 \mathrm{~F}$ isoforms along the nephron in mice. Am J Physiol Renal Physiol. 2003 Jan;284(1):F95-102.

33 Wu CC, Mei S, Cheng J, Ding Y, Weidenhammer A, Garcia V, et al. Androgen-sensitive hypertension associates with upregulated vascular CYP4A12-20-HETE synthase. J Am Soc Nephrol. 2013 Jul;24(8):1288-96.

34 Gangadhariah MH, Luther JM, Garcia V, Paueksakon P, Zhang MZ, Hayward SW, et al. Hypertension is a major contributor to 20-hydroxyeicosatetraenoic acid-mediated kidney injury in diabetic nephropathy. J Am Soc Nephrol. 2015 Mar;26(3):597-610.

35 Nakagawa K, Holla VR, Wei Y, Wang WH, Gatica A, Wei S, et al. Salt-sensitive hypertension is associated with dysfunctional Cyp4a10 gene and kidney epithelial sodium channel. J Clin Invest. 2006 Jun;116(6): 1696-702.

36 Zhou Y, Huang H, Chang HH, Du J, Wu JF, Wang CY, et al. Induction of renal 20-hydroxyeicosatetraenoic acid by clofibrate attenuates high-fat diet-induced hypertension in rats. J Pharmacol Exp Ther. 2006 Apr; 317(1):11-8.

37 Riazi S, Khan O, Tiwari S, Hu X, Ecelbarger $\mathrm{CA}$. Rosiglitazone regulates $\mathrm{ENaC}$ and $\mathrm{Na}-\mathrm{K}$ $2 \mathrm{Cl}$ cotransporter (NKCC2) abundance in the obese Zucker rat. Am J Nephrol. 2006;26(3): 245-57.

38 Chraïbi A, Renauld S. PPAR $\gamma$-induced stimulation of amiloride-sensitive sodium current in renal collecting duct principal cells is serum and insulin dependent. Cell Physiol Biochem. 2014;33(3):581-93.

39 Song J, Knepper MA, Hu X, Verbalis JG, Ecelbarger CA. Rosiglitazone activates renal sodium- and water- reabsorptive pathways and lowers blood pressure in normal rats. J Pharmacol Exp Ther. 2004 Feb;308(2):426-33.

40 Vallon V, Hummler E, Rieg T, Pochynyuk O, Bugaj V, Schroth J, et al. Thiazolidinedioneinduced fluid retention is independent of collecting duct alphaENaC activity. J Am Soc Nephrol. 2009 Apr;20(4):721-9.

41 da Silva CA, de Bragança AC, Shimizu MH, Sanches TR, Fortes MA, Giorgi RR, et al. Rosiglitazone prevents sirolimus-induced hypomagnesemia, hypokalemia, and downregulation of NKCC2 protein expression. Am J Physiol Renal Physiol. 2009 Oct;297(4):F91622.

42 Renauld S, Tremblay K, Ait-Benichou S, Simoneau-Roy M, Garneau H, Staub O, et al. Stimulation of $\mathrm{ENaC}$ activity by rosiglitazone is PPAR $\gamma$-dependent and correlates with SGK1 expression increase. J Membr Biol. 2010 Aug;236(3):259-70.
43 Vallon V, Lang F. New insights into the role of serum- and glucocorticoid-inducible kinase SGK1 in the regulation of renal function and blood pressure. Curr Opin Nephrol Hypertens. 2005 Jan;14(1):59-66.

44 Ishizuka T, Ito O, Tan L, Ogawa S, Kohzuki $\mathrm{M}$, Omata K, et al. Regulation of cytochrome P-450 4A activity by peroxisome proliferatoractivated receptors in the rat kidney. Hypertens Res. 2003 Nov;26(11):929-36.

45 Bishop-Bailey D. Peroxisome proliferator-activated receptors in the cardiovascular system. Br J Pharmacol. 2000 Mar;129(5):82334.

46 Ito O, Nakamura Y, Tan L, Ishizuka T, Sasaki $\mathrm{Y}$, Minami N, et al. Expression of cytochrome P-450 4 enzymes in the kidney and liver: regulation by PPAR and species-difference between rat and human. Mol Cell Biochem. 2006 Mar;284(1-2):141-8.

47 Fang X, Dillon JS, Hu S, Harmon SD, Yao J, Anjaiah S, et al. 20-Carboxy-arachidonic acid is a dual activator of peroxisome proliferatoractivated receptors alpha and gamma. Prostaglandins Other Lipid Mediat. 2007 Jan;82(14):175-84.

48 Lee DL, Wilson JL, Duan R, Hudson T, ElMarakby A. Peroxisome proliferator-activated receptor- $\alpha$ activation decreases mean arterial pressure, plasma interleukin-6, and COX2 while increasing renal CYP4A expression in an acute model of DOCA-salt hypertension. PPAR Res. 2011;2011:502631.

49 Han T, Lv Y, Wang S, Hu T, Hong H, Fu Z. PPAR $\gamma$ overexpression regulates cholesterol metabolism in human L02 hepatocytes. J Pharmacol Sci. 2019 Jan;139(1):1-8.

50 Deng J, Guo L, Wu B. Circadian regulation of hepatic cytochrome $\mathrm{P} 4502 \mathrm{a} 5$ by peroxisome proliferator-activated receptor $\gamma$. Drug Metab Dispos. 2018 Nov;46(11):1538-45.

51 Zhang F, Li J, Na S, Wu J, Yang Z, Xie X, et al. The involvement of PPARs in the selective regulation of brain CYP2D by growth hormone. Neuroscience. 2018 May 21;379:115-25.

52 Yi M, Shin JG, Lee SJ. Expression of CYP4V2 in human THP1 macrophages and its transcriptional regulation by peroxisome proliferator-activated receptor gamma. Toxicol Appl Pharmacol. 2017 Sep 1;330:100-6.

53 Ng VY, Huang Y, Reddy LM, Falck JR, Lin ET, Kroetz DL. Cytochrome P450 eicosanoids are activators of peroxisome proliferator-activated receptor alpha. Drug Metab Dispos. 2007 Jul;35(7):1126-34.

$54 \mathrm{Na} \mathrm{S}$, Li J, Zhang H, Li Y, Yang Z, Zhong Y, et al. The induction of cytochrome P450 2E1 by ethanol leads to the loss of synaptic proteins via PPARa down-regulation. Toxicology. 2017 Jun 15;385:18-27.

55 Riazi S, Khan O, Hu X, Ecelbarger CA. Aldosterone infusion with high- $\mathrm{NaCl}$ diet increases blood pressure in obese but not lean Zucker rats. Am J Physiol Renal Physiol. 2006 Sep; 291(3):F597-605. 
56 Madala Halagappa VK, Tiwari S, Riazi S, Hu $\mathrm{X}$, Ecelbarger CM. Chronic candesartan alters expression and activity of NKCC2, NCC, and $\mathrm{ENaC}$ in the obese Zucker rat. Am J Physiol Renal Physiol. 2008 May;294(5): F1222-31.

57 Bickel CA, Knepper MA, Verbalis JG, Ecelbarger CA. Dysregulation of renal salt and water transport proteins in diabetic Zucker rats. Kidney Int. 2002 Jun;61(6):2099-110.

58 Zhou L, Liu G, Jia Z, Yang KT, Sun Y, Kakizoe $\mathrm{Y}$, et al. Increased susceptibility of $\mathrm{db} / \mathrm{db}$ mice to rosiglitazone-induced plasma volume expansion: role of dysregulation of renal water transporters. Am J Physiol Renal Physiol. 2013 Nov 15;305(10):F1491-7.

59 Sakai K, Yamazaki O, Ishizawa K, Tamura Y, Wang Q, Ueno M, et al. Upregulation of renal
$\mathrm{Na}-\mathrm{K}-2 \mathrm{Cl}$ cotransporter 2 in obese diabetes mellitus via a vasopressin receptor 2-dependent pathway. Biochem Biophys Res Commun. 2020 Apr 9;524(3):710-5.

60 Carolan E, Hogan AE, Corrigan M, Gaotswe G, O'Connell J, Foley N, et al. The impact of childhood obesity on inflammation, innate immune cell frequency, and metabolic microRNA expression. J Clin Endocrinol Metab. 2014 Mar;99(3):E474-8.

61 Keustermans GC, Kofink D, Eikendal A, de Jager W, Meerding J, Nuboer R, et al. Monocyte gene expression in childhood obesity is associated with obesity and complexity of atherosclerosis in adults. Sci Rep. 2017 Dec 4; 7(1):16826

62 Liu Y, Ji Y, Li M, Wang M, Yi X, Yin C, et al. Integrated analysis of long noncoding RNA and mRNA expression profile in children with obesity by microarray analysis. Sci Rep. 2018 Jun $8 ; 8(1): 8750$.

63 Martino F, Magenta A, Pannarale G, Martino E, Zanoni C, Perla FM, et al. Epigenetics and cardiovascular risk in childhood. J Cardiovasc Med. 2016 Aug;17(8):539-46.

64 Ouyang S, Tang R, Liu Z, Ma F, Li Y, Wu J. Characterization and predicted role of microRNA expression profiles associated with early childhood obesity. Mol Med Rep. 2017 Oct;16(4):3799-806.

65 Khan O, Riazi S, Hu X, Song J, Wade JB, Ecelbarger CA. Regulation of the renal thiazidesensitive $\mathrm{Na}-\mathrm{Cl}$ cotransporter, blood pressure, and natriuresis in obese Zucker rats treated with rosiglitazone. Am J Physiol Renal Physiol. 2005 Aug;289(2):F442-50. 